ANNALES

POLONICI MATHEMATICI

$80(2003)$

\title{
Normal families and shared values of meromorphic functions
}

by Mingliang FAng (Nanjing) and LAWRence Zalcman (Ramat-Gan)

To Professor Józef Siciak, with admiration and friendship

\begin{abstract}
Let $\mathcal{F}$ be a family of meromorphic functions on a plane domain $D$, all of whose zeros are of multiplicity at least $k \geq 2$. Let $a, b, c$, and $d$ be complex numbers such that $d \neq b, 0$ and $c \neq a$. If, for each $f \in \overline{\mathcal{F}}, f(z)=a \Leftrightarrow f^{(k)}(z)=b$, and $f^{(k)}(z)=d \Rightarrow$ $f(z)=c$, then $\mathcal{F}$ is a normal family on $D$. The same result holds for $k=1$ so long as $b \neq(m+1) d, m=1,2, \ldots$
\end{abstract}

1. Introduction. Let $f$ and $g$ be meromorphic functions on a domain $D$ in $\mathbb{C}$, and let $a$ and $b$ be complex numbers. If $g(z)=b$ whenever $f(z)=a$, we write $f(z)=a \Rightarrow g(z)=b$. If $f(z)=a \Rightarrow g(z)=b$ and $g(z)=b \Rightarrow f(z)=a$, we write $f(z)=a \Leftrightarrow g(z)=b$. If $f(z)=a \Leftrightarrow g(z)=a$, then we say that $f$ and $g$ share $a$ in $D$.

Mues and Steinmetz [11] proved

THEOREM A. Let $f$ be a nonconstant meromorphic function, and let $a_{1}$, $a_{2}$, and $a_{3}$ be distinct complex numbers. If $f$ and $f^{\prime}$ share $a_{1}, a_{2}$, and $a_{3}$, then $f \equiv f^{\prime}$.

Schwick [15] discovered a connection between normality criteria and shared values. He proved

THEOREM B. Let $\mathcal{F}$ be a family of meromorphic functions in a domain $D$, and let $a_{1}, a_{2}$, and $a_{3}$ be distinct complex numbers. If , for each $f \in \mathcal{F}$, $f$ and $f^{\prime}$ share $a_{1}, a_{2}$, and $a_{3}$ in $D$, then $\mathcal{F}$ is normal in $D$.

2000 Mathematics Subject Classification: Primary 30D45.

Key words and phrases: normal families, shared values.

Research of the first author supported by the National Natural Science Foundation of China (grant no. 10071038) and by the Fred and Barbara Kort Sino-Israel Post Doctoral Fellowship Program at Bar-Ilan University.

Research of the second author supported by the German-Israeli Foundation for Scientific Research and Development, G.I.F. grant no. G-643-117.6/1999. 
This result has undergone various extensions [12], [17], [18], culminating in the following result of Pang and Zalcman [13].

Theorem C. Let $\mathcal{F}$ be a family of meromorphic functions in a domain $D$; and let $a, b$, and $c$ be complex numbers such that $b \neq a$ and $c \neq 0$. If, for each $f \in \mathcal{F}, f(z)=0 \Leftrightarrow f^{\prime}(z)=a$, and $f(z)=c \Leftrightarrow f^{\prime}(z)=b$, then $\mathcal{F}$ is normal in $D$.

It is natural to ask what can be said if $f^{\prime}$ is replaced by $f^{(k)}$ for $k \geq 2$ in the above theorems. Frank and Schwick observed that while Theorem A extends in a natural fashion when $f^{\prime}$ is replaced by $f^{(k)}[6]$, Theorem B does not admit such an extension [7]. Chen and Fang [4] proved

TheOREM D. Let $\mathcal{F}$ be a family of meromorphic functions in a domain $D$; let $k \geq 2$ be an integer; and let $a, b$, and $c$ be complex numbers such that $b \neq a$. If , for each $f \in \mathcal{F}, f$ and $f^{(k)}$ share $a$ and $b$ in $D$, and all zeros of $f-c$ have multiplicity at least $k+1$, then $\mathcal{F}$ is normal in $D$.

In this paper, we extend Theorem $\mathrm{C}$ as follows.

THEOREM 1. Let $\mathcal{F}$ be a family of meromorphic functions in a domain $D$; let $k$ be a positive integer; and let $a, b, c$, and $d$ be complex numbers such that $b \neq a, 0$ and $c \neq 0$. If , for each $f \in \mathcal{F}$, all zeros of $f-d$ have multiplicity at least $k, f(z)=0 \Leftrightarrow f^{(k)}(z)=a$, and $f^{(k)}(z)=b \Rightarrow f(z)=c$, then $\mathcal{F}$ is normal in $D$ for $k \geq 2$, and for $k=1$ so long as $a \neq(m+1) b$, $m=1,2, \ldots$.

As a consequence, we obtain the following sharpening of Theorem D.

COROLlary. Let $\mathcal{F}$ be a family of meromorphic functions in a domain $D$; let $k \geq 2$ be an integer; and let $a, b$, and $c$ be complex numbers such that $b \neq a$. If , for each $f \in \mathcal{F}, f$ and $f^{(k)}$ share $a$ and $b$ in $D$, and all zeros of $f-c$ have multiplicity at least $k$, then $\mathcal{F}$ is normal in $D$.

Proof. Since $a \neq b$, we may assume that $b \neq 0$. Consider the family of functions $\mathcal{G}=\{f-a: f \in \mathcal{F}\}$. For each $g \in \mathcal{G}$, all zeros of $g-(c-a)$ have multiplicity at least $k$. Further, if $g \in \mathcal{G}$, then $g(z)=0 \Leftrightarrow g^{(k)}(z)=a$, and $g(z)=b-a \Leftrightarrow g^{(k)}(z)=b$. By Theorem $1, \mathcal{G}$ is normal on $D$; and hence $\mathcal{F}$ is normal on $D$.

Example 1. Consider the family $\mathcal{F}=\left\{a\left(e^{n z}-1\right) / n: n=1,2, \ldots\right\}$ on $D=\{z:|z|<1\}$. Then, for every $f \in \mathcal{F}, f(z)=0 \Leftrightarrow f^{\prime}(z)=a$, and $f^{\prime}(z) \neq 0$ (and hence $f^{\prime}(z)=0 \Rightarrow f(z)=c$ for any $c$ ). But $\mathcal{F}$ is not normal in $D$. This shows that $b \neq 0$ is necessary in Theorem 1 when $k=1$. For $k \geq 2$, Theorem 1 actually holds even when $b=0$. However, we shall not prove that here. 
EXAMPLE 2. Let $a$ and $b$ be two nonzero numbers such that $a=(m+1) b$, where $m$ is a positive integer. Set

$$
f_{n}(z)=b\left(z-\frac{1}{n}\right)+\frac{1}{m(n z-1)^{m}}, \quad n=1,2, \ldots,
$$

and let $\mathcal{F}=\left\{f_{n}\right\}, D=\{z:|z|<1\}$. Then

$$
f_{n}^{\prime}(z)=b-\frac{n}{(n z-1)^{m+1}} .
$$

Clearly, for every $f \in \mathcal{F}, f(z)=0 \Leftrightarrow f^{\prime}(z)=a$, and $f^{\prime}(z) \neq b$ (hence $\left.f^{\prime}(z)=b \Rightarrow f(z)=c\right)$. But $\mathcal{F}$ is not normal in $D$. This means that $a \neq$ $(m+1) b(m=1,2, \ldots)$ is necessary in Theorem 1 when $k=1$.

EXAMPLE 3. Fix $k$ and let $\left\{\omega_{1}, \ldots, \omega_{k}\right\}$ be the $k$ th roots of unity (with $\left.\omega_{k}=1\right)$. Any function of the form

$$
F(z)=\sum_{j=1}^{k} c_{j} e^{\omega_{j} z}
$$

clearly satisfies $F^{(k)} \equiv F$. The $k \times k$ Vandermonde determinant defined by $\omega_{j}, 1 \leq j \leq k$, does not vanish. Hence, solving $k$ linear equations in $k$ unknowns, we may choose the $c_{j}$ so that the first $k-1$ Taylor coefficients of $F$ vanish at the origin, i.e., so that $F$ has a zero of exact order $k-1$ at 0 . Let $D=\{z:|z|<1\}$, and set $f_{n}(z)=n F(z), n=1,2, \ldots$ Let $\mathcal{F}=\left\{f_{n}\right\}$; then $\mathcal{F}$ is a family of holomorphic functions on $D$. Obviously, for each $f \in \mathcal{F}, f^{(k)} \equiv f$, so $f$ and $f^{(k)}$ share every complex value in $D$. But $\mathcal{F}$ is not normal in $D$. This shows that the requirement of multiplicity $k$ in Theorem 1 cannot be dropped in general.

EXAMPLE 4. Theorem 1 does not hold if the requirement that $f^{(k)}(z)=$ $b \Rightarrow f(z)=c$ is replaced by $f(z)=c \Rightarrow f^{(k)}(z)=b$. Indeed, set

$$
f_{n}(z)=\frac{(n z)^{2}}{(n z)^{2}-1}, \quad n=1,2, \ldots,
$$

and let $\mathcal{F}=\left\{f_{n}\right\}, D=\{z:|z|<1\}$. Then

$$
f_{n}^{\prime}(z)=\frac{-2 n^{2} z}{\left[(n z)^{2}-1\right]^{2}} .
$$

Obviously, if $f \in \mathcal{F}$, then $f$ and $f^{\prime}$ vanish only at 0 ; also, $f(z) \neq 1$. Thus, if we choose $k=1, a=0$, and $c=1$, we have $f(z)=0 \Leftrightarrow f^{\prime}(z)=0$, and $f(z)=1 \Rightarrow f^{\prime}(z)=b$ for any $b$ (since $f(z) \neq 1$ ). However, $\mathcal{F}$ is not normal on $D$.

THEOREM 2. Let $f$ be a transcendental meromorphic function, $k \geq 2$ an integer, and $a \in \mathbb{C}$. If all zeros of $f$ have multiplicity at least $k$ and $f(z)=0 \Leftrightarrow f^{(k)}(z)=a$, then $f^{(k)}$ takes on each nonzero finite value $b$ infinitely many times. 
2. Some lemmas. For the proofs of our theorems, we need the following lemmas.

Lemma 1 ([14, Lemma 2]). Let $\mathcal{F}$ be a family of functions meromorphic on the unit disc, all of whose zeros have multiplicity at least $k$, and suppose that there exists $A \geq 1$ such that $\left|f^{(k)}(z)\right| \leq A$ whenever $f(z)=0$. Then if $\mathcal{F}$ is not normal, there exist, for each $0 \leq \alpha \leq k$,

(a) a number $0<r<1$;

(b) points $z_{n},\left|z_{n}\right|<r$;

(c) functions $f_{n} \in \mathcal{F}$; and

(d) positive numbers $\varrho_{n} \rightarrow 0$

such that $\varrho_{n}^{-\alpha} f_{n}\left(z_{n}+\varrho_{n} \zeta\right)=g_{n}(\zeta) \rightarrow g(\zeta)$ locally uniformly with respect to the spherical metric, where $g$ is a nonconstant meromorphic function on $\mathbb{C}$, all of whose zeros have multiplicity at least $k$, such that $g^{\#}(\zeta) \leq g^{\#}(0)=$ $k A+1$. In particular, $g$ has order at most 2 .

Here, as usual, $g^{\#}(\zeta)=\left|g^{\prime}(\zeta)\right| /\left(1+|g(\zeta)|^{2}\right)$ is the spherical derivative.

REMARK. That all zeros of $g$ have multiplicity at least $k$ is immediate from the argument principle. That $g$ has order at most 2 follows easily from the fact that $g^{\#}$ is bounded; cf. [19, p. 217]. For $0 \leq \alpha<k$, the hypothesis on $f^{(k)}(z)$ can be dropped, and $k A+1$ can be replaced by an arbitrary positive constant.

Lemma 2 ([3, Corollary 3]). Let $g$ be a meromorphic function with finite order. If $g$ has only finitely many critical values, then it has only finitely many asymptotic values.

Lemma 3 ([1, Lemma 2]; cf. [2, Lemma 3]). Let $g$ be a transcendental meromorphic function such that $g(0) \neq \infty$ and the set of finite critical and asymptotic values of $g$ is bounded. Then there exists $R>0$ such that

$$
\left|g^{\prime}(z)\right| \geq \frac{|g(z)|}{2 \pi|z|} \log \frac{|g(z)|}{R}
$$

for all $z \in \mathbb{C} \backslash\{0\}$ which are not poles of $g$.

Lemma 4 ([8, Theorem 3], [9, Corollary to Theorem 3.5]). Let $f$ be a transcendental meromorphic function, and let $b$ be a nonzero value. Then, for each positive integer $k$, either $f$ or $f^{(k)}-b$ has infinitely many zeros.

LEMma 5. Let $f$ be a transcendental meromorphic function of finite order in the complex plane, $k$ a positive integer, and $a$ and $b \neq 0$ complex numbers. If all zeros of $f$ have multiplicity at least $k$ and $f(z)=0 \Leftrightarrow f^{(k)}(z)=a$, then $f^{(k)}-b$ has infinitely many zeros.

Proof. We consider two cases. 
CASE 1: $f$ has only finitely many zeros. In this case, $f^{(k)}-b$ has infinitely many zeros by Lemma 4 .

CASE 2: $f$ has infinitely many zeros $z_{1}, z_{2}, \ldots$ We define $g(z)=$ $f^{(k-1)}(z)-b z$; then $g^{\prime}(z)=f^{(k)}(z)-b$. We have to show that $g^{\prime}$ has infinitely many zeros. Suppose that $g^{\prime}$ has only finitely many zeros; then $g$ has finitely many critical values. Hence, by Lemma $2, g$ has only finitely many asymptotic values. Without loss of generality, we may assume that $f(0) \neq \infty$ (and hence $g(0) \neq \infty$ ). Then by Lemma 3 we have

$$
\frac{\left|z_{j} g^{\prime}\left(z_{j}\right)\right|}{\left|g\left(z_{j}\right)\right|} \geq \frac{1}{2 \pi} \log \frac{\left|g\left(z_{j}\right)\right|}{R}=\frac{1}{2 \pi} \log \frac{b\left|z_{j}\right|}{R} .
$$

In particular,

$$
\frac{\left|z_{j} g^{\prime}\left(z_{j}\right)\right|}{\left|g\left(z_{j}\right)\right|} \rightarrow \infty \quad \text { as } j \rightarrow \infty
$$

On the other hand,

$$
\frac{\left|z_{j} g^{\prime}\left(z_{j}\right)\right|}{\left|g\left(z_{j}\right)\right|}=\left|\frac{a-b}{b}\right|,
$$

a contradiction. It follows that $g^{\prime}(z)=f^{(k)}(z)-b$ has infinitely many zeros. This completes the proof of Lemma 5.

Lemma 6 ([16, Lemma 8]). Let $f(z)=a_{n} z^{n}+a_{n-1} z^{n-1}+\ldots+a_{0}+$ $q(z) / p(z)$ where $a_{0}, a_{1}, \ldots, a_{n}$ are constants with $a_{n} \neq 0$ and $p$ and $q$ are two coprime polynomials, neither of which vanishes identically, with $\operatorname{deg} q<\operatorname{deg} p ;$ and let $k$ be a positive integer. If $f^{(k)}(z) \neq 1$, then

$$
f(z)=\frac{z^{k}}{k !}+\ldots+a_{0}+\frac{1}{(\alpha z+\beta)^{m}} .
$$

Here $\alpha \neq 0$ and $\beta$ are constants and $m$ is a positive integer.

Lemma 7. Let $f$ be a meromorphic function of finite order, $a$ and $b \neq 0$ distinct complex numbers, and $k \geq 2$ a positive integer. If all zeros of $f$ have multiplicity at least $k, f(z)=0 \Leftrightarrow f^{(k)}(z)=a$, and $f^{(k)}(z) \neq b$, then $f$ is constant.

Proof. By Lemma $5, f$ is a rational function. We assume $f(z)=a_{n} z^{n}+$ $a_{n-1} z^{n-1}+\ldots+a_{0}+q(z) / p(z)$, where $a_{0}, a_{1}, \ldots, a_{n}$ are constants with $a_{n} \neq 0, q$ and $p$ are two coprime polynomials with $\operatorname{deg} q<\operatorname{deg} p$, and $n$ is a positive integer. Without loss of generality, we assume that $b=1$. Suppose that $q$ does not vanish identically. Then by Lemma 6 ,

$$
f(z)=\frac{1}{k !} z^{k}+\ldots+a_{0}+\frac{1}{(\alpha z+\beta)^{m}}, \quad f^{(k)}(z)=1+\frac{A}{(\alpha z+\beta)^{k+m}},
$$

where $A \neq 0, \alpha \neq 0$ and $\beta$ are constants. Since the zeros of $f$ all have multiplicity at least $k$, the set $\{z \in \mathbb{C}: f(z)=0\}$ has at most $(k+m) / k$ 
distinct elements, while the set $\left\{z \in \mathbb{C}: f^{(k)}(z)=a\right\}$ has $k+m$ distinct elements. This contradicts the assumptions that $f(z)=0 \Leftrightarrow f^{(k)}(z)=a$ and $k \geq 2$.

It follows that $f$ is a polynomial. In this case, one checks easily that $f$ is constant. The lemma is proved.

Using Lemmas 5 and 6 , we obtain, after a simple calculation, the following result.

Lemma 8 (cf. [13, Lemma 6]). Let $f$ be a nonconstant meromorphic function of finite order, and let $a$ and $b \neq 0$ be complex numbers. If $f(z)=0$ $\Leftrightarrow f^{\prime}(z)=a$, and $f^{\prime}(z) \neq b$ in $\mathbb{C}$, then

$$
f(z)=b(z-d)+\frac{A}{m(z-d)^{m}}, \quad a=(m+1) b,
$$

for some $d \in \mathbb{C}$ and some positive integer $m$.

Lemma 9 ([5], [10]; cf. [2]). Let $f$ be a nonconstant meromorphic function on the plane and $k \geq 2$ a positive integer. Suppose that $f(z) \neq 0$ and $f^{(k)}(z) \neq 0$ for all $z \in \mathbb{C}$. Then either $f(z)=e^{A z+B}$ or $f(z)=\frac{1}{(A z+B)^{m}}$, where $A \neq 0$ and $B$ are constants and $m$ is a positive integer.

3. Proof of Theorem 1. We may assume that $D=\Delta$, the unit disc. Suppose that $\mathcal{F}$ is not normal on $\Delta$. We consider separately the cases $d=0$ and $d \neq 0$.

Case I. Suppose $d=0$. Then by Lemma 1 , we can find $f_{n} \in \mathcal{F}, z_{n} \in \Delta$, and $\varrho_{n} \rightarrow 0^{+}$such that $g_{n}(\zeta)=\varrho_{n}^{-k} f_{n}\left(z_{n}+\varrho_{n} \zeta\right)$ converges locally uniformly with respect to the spherical metric to a nonconstant meromorphic function $g$ on $\mathbb{C}$, all of whose zeros have multiplicity at least $k$, which satisfies $g^{\#}(\zeta) \leq$ $g^{\#}(0)=k(|a|+1)+1$. In particular, $g$ is of order at most 2 .

We claim that

(i) $g(\zeta)=0 \Leftrightarrow g^{(k)}(\zeta)=a$, and

(ii) $g^{(k)}(\zeta) \neq b$ on $\mathbb{C}$.

Suppose that $g\left(\zeta_{0}\right)=0$. Then by the Hurwitz Theorem, there exist $\zeta_{n}$, $\zeta_{n} \rightarrow \zeta_{0}$, such that (for $n$ sufficiently large) $0=g_{n}\left(\zeta_{n}\right)=f_{n}\left(z_{n}+\varrho_{n} \zeta_{n}\right) / \varrho_{n}^{k}$. Thus $f_{n}\left(z_{n}+\varrho_{n} \zeta_{n}\right)=0$. Hence $f_{n}^{(k)}\left(z_{n}+\varrho_{n} \zeta_{n}\right)=a$, so that $g_{n}^{(k)}\left(\zeta_{n}\right)=$ $f_{n}^{(k)}\left(z_{n}+\varrho_{n} \zeta_{n}\right)=a$. Since $g^{(k)}\left(\zeta_{0}\right)=\lim _{n \rightarrow \infty} g_{n}^{(k)}\left(\zeta_{n}\right)=a$, we have shown that $g(\zeta)=0 \Rightarrow g^{(k)}(\zeta)=a$.

Suppose now that $g^{(k)}\left(\zeta_{0}\right)=a$. We claim that $g^{(k)} \not \equiv a$. Indeed, if $a=0$, $g$ would be a polynomial of degree less than $k$ and so could not have zeros of multiplicity at least $k$. If $a \neq 0, g$ must be a polynomial of exact degree $k$. Since each zero of $g$ has multiplicity at least $k, g$ must have a single zero 
$\zeta_{1}$ of multiplicity $k$, so that $g(\zeta)=a\left(\zeta-\zeta_{1}\right)^{k} / k$ !. A simple calculation then shows that

$$
g^{\#}(0) \leq \begin{cases}k / 2 & \text { if }\left|\zeta_{1}\right| \geq 1 \\ |a| & \text { if }\left|\zeta_{1}\right|<1\end{cases}
$$

so that $g^{\#}(0)<k(|a|+1)+1$, a contradiction. Since $g^{(k)}\left(\zeta_{0}\right)=a$ but $g^{(k)} \not \equiv a$, there exist $\zeta_{n}, \zeta_{n} \rightarrow \zeta_{0}$, such that $f_{n}^{(k)}\left(z_{n}+\varrho_{n} \zeta_{n}\right)=g_{n}^{(k)}\left(\zeta_{n}\right)=a$ for $n$ sufficiently large. It follows that $f_{n}\left(z_{n}+\varrho_{n} \zeta_{n}\right)=0$, so that $g_{n}\left(\zeta_{n}\right)=$ $f_{n}\left(z_{n}+\varrho_{n} \zeta_{n}\right) / \varrho_{n}^{k}=0$. Since $g\left(\zeta_{0}\right)=\lim _{n \rightarrow \infty} g_{n}\left(\zeta_{n}\right)=0$, we have shown that $g^{(k)}(\zeta)=a \Rightarrow g(\zeta)=0$.

This proves (i).

Next we prove (ii). Suppose $g^{(k)}\left(\zeta_{0}\right)=b$. Then $g\left(\zeta_{0}\right) \neq \infty$. Further $g^{(k)} \not \equiv b$, since that would imply $g(\zeta)=b\left(\zeta-\zeta_{1}\right)^{k} / k$ !, which is inconsistent with (i). Thus, by the Hurwitz Theorem, there exist $\zeta_{n}, \zeta_{n} \rightarrow \zeta_{0}$, such that (for large $n) g_{n}^{(k)}\left(\zeta_{n}\right)=f_{n}^{(k)}\left(z_{n}+\varrho_{n} \zeta_{n}\right)=b$. Since $f_{n}^{(k)}(z)=b \Rightarrow f_{n}(z)=c$, we have $f_{n}\left(z_{n}+\varrho_{n} \zeta_{n}\right)=c$ and $g_{n}\left(\zeta_{n}\right)=f_{n}\left(z_{n}+\varrho_{n} \zeta_{n}\right) / \varrho_{n}^{k}=c / \varrho_{n}^{k} \rightarrow \infty$, which contradicts $\lim _{n \rightarrow \infty} g_{n}\left(\zeta_{n}\right)=g\left(\zeta_{0}\right) \neq \infty$. That proves (ii).

If $k \geq 2, g$ is constant by Lemma 7 , a contradiction. If $k=1$, then by Lemma 8,

$$
g(\zeta)=b(\zeta-d)+\frac{A}{m(\zeta-d)^{m}}, \quad a=(m+1) b,
$$

for some positive integer $m$, a possibility that is ruled out explicitly in the hypothesis of the theorem. Thus $\mathcal{F}$ is normal on $D$.

CASE II. Suppose now that $d \neq 0$. We may assume that $k \geq 2$. By Lemma 1 , we can find $f_{n} \in \mathcal{F}, z_{n} \in \Delta$, and $\varrho_{n} \rightarrow 0^{+}$such that $g_{n}(\zeta)=$ $f_{n}\left(z_{n}+\varrho_{n} \zeta\right)-d$ converges locally uniformly with respect to the spherical metric to a nonconstant meromorphic function $g$ on $\mathbb{C}$, all of whose zeros have multiplicity at least $k$.

We claim that

(iii) $g^{(k)}(\zeta) \neq 0$ on $\mathbb{C}$, and

(iv) $g(\zeta) \neq-d$ on $\mathbb{C}$.

Suppose that $g^{(k)}\left(\zeta_{0}\right)=0$. Clearly $g^{(k)} \not \equiv 0$, for otherwise $g$ would be a polynomial of degree less than $k$, and so could not have zeros of multiplicity at least $k$. Hence, since $g_{n}^{(k)}(\zeta)-\varrho_{n}^{k} a \rightarrow g^{(k)}(\zeta)$ on a neighborhood of $\zeta_{0}$, there exist $\zeta_{n}, \zeta_{n} \rightarrow \zeta_{0}$, such that (for $n$ sufficiently large)

$$
0=g^{(k)}\left(\zeta_{0}\right)=g_{n}^{(k)}\left(\zeta_{n}\right)-\varrho_{n}^{k} a=\varrho_{n}^{k}\left[f_{n}^{(k)}\left(z_{n}+\varrho_{n} \zeta_{n}\right)-a\right] .
$$

Thus $f_{n}^{(k)}\left(z_{n}+\varrho_{n} \zeta_{n}\right)=a$, so that $f_{n}\left(z_{n}+\varrho_{n} \zeta_{n}\right)=0$. It follows that $g_{n}\left(\zeta_{n}\right)=$ $f_{n}\left(z_{n}+\varrho_{n} \zeta_{n}\right)-d=-d$, and so $g\left(\zeta_{0}\right)=\lim _{n \rightarrow \infty} g_{n}\left(\zeta_{n}\right)=-d$. 
In a similar fashion, considering $g_{n}^{(k)}(\zeta)-\varrho_{n}^{k} b$ instead of $g_{n}^{(k)}(\zeta)-\varrho_{n}^{k} a$, we obtain $g\left(\zeta_{0}\right)=c-d$. Thus $c=0$, contrary to assumption. This completes the proof of (iii).

Finally, we prove (iv). Suppose that $g\left(\zeta_{0}\right)=-d$. Then there exist $\zeta_{n}$, $\zeta_{n} \rightarrow \zeta_{0}$, such that (for $n$ sufficiently large) $-d=g\left(\zeta_{0}\right)=g_{n}\left(\zeta_{n}\right)=$ $f_{n}\left(z_{n}+\varrho_{n} \zeta_{n}\right)-d$. Thus $f_{n}\left(z_{n}+\varrho_{n} \zeta_{n}\right)=0$, and hence $f_{n}^{(k)}\left(z_{n}+\varrho_{n} \zeta_{n}\right)=a$. It follows that $g_{n}^{(k)}\left(\zeta_{n}\right)=\varrho_{n}^{k} f_{n}^{(k)}\left(z_{n}+\varrho_{n} \zeta_{n}\right) \rightarrow 0$. Therefore, $g^{(k)}\left(\zeta_{0}\right)=$ $\lim _{n \rightarrow \infty} g_{n}^{(k)}\left(\zeta_{n}\right)=0$. But this contradicts (iii). That proves (iv).

Now by Lemma 9, either $g(\zeta)=-d+e^{A \zeta+B}$ or $g(\zeta)=-d+1 /(A z+B)^{m}$, where $A \neq 0$ and $B$ are constants and $m$ is a positive integer. In either case, $g$ has a nonempty set of zeros (it is here that we use the assumption $d \neq 0$ ), all of which are obviously simple. This contradicts the fact that all zeros of $g$ have multiplicity at least $k \geq 2$. Thus, in Case II also, $\mathcal{F}$ is normal. This completes the proof of Theorem 1 .

4. Proof of Theorem 2. From Theorem 1, we obtain the following result, which will be used in the proof of Theorem 2 .

Lemma 10. Let $\mathcal{F}$ be a family of meromorphic functions in a domain $D$; let $k \geq 2$ be an integer; and let $a$ and $b \neq 0$ be distinct complex numbers. If, for each $f \in \mathcal{F}$, all zeros of $f$ have multiplicity at least $k, f(z)=0 \Leftrightarrow$ $f^{(k)}(z)=a$, and $f^{(k)}(z) \neq b$, then $\mathcal{F}$ is normal in $D$.

Now we prove Theorem 2 .

In case $b=a \neq 0$, the theorem follows at once from Lemma 4. Suppose then that $b \neq a, 0$. If $f$ has finite order, the theorem then follows from Lemma 5. So suppose that $f$ has infinite order. Then $f^{\#}$ is unbounded on $\mathbb{C}$, so there exist $w_{n} \rightarrow \infty$ such that $f^{\#}\left(w_{n}\right) \rightarrow \infty$. Let $f_{n}(z)=f\left(z+w_{n}\right)$ and consider the family $\mathcal{F}=\left\{f_{n}\right\}$ on the unit disc $\Delta$. Clearly, for each $n$, all zeros of $f_{n}$ have multiplicity at least $k$ and $f_{n}(z)=0 \Leftrightarrow f_{n}^{(k)}(z)=a$. Since $f_{n}^{\#}(0)=f^{\#}\left(w_{n}\right) \rightarrow \infty$, no infinite subfamily of $\mathcal{F}$ is normal on $\Delta$. Suppose now that $f^{(k)}(z)=b$ has only finitely many solutions. Then, since $w_{n} \rightarrow \infty$, there exists $N$ such that no function in $\mathcal{F}_{N}=\left\{f_{n}: n \geq N\right\}$ takes on the value $b$ in $\Delta$. By Lemma $10, \mathcal{F}_{N}$ is normal on $\Delta$, a contradiction.

\section{References}

[1] W. Bergweiler, On the zeros of certain homogeneous differential polynomials, Arch. Math. (Basel) 64 (1995), 199-202.

[2] -, Normality and exceptional values of derivatives, Proc. Amer. Math. Soc. 129 (2001), 121-129.

[3] W. Bergweiler and A. Eremenko, On the singularities of the inverse to a meromorphic function of finite order, Rev. Mat. Iberoamericana 11 (1995), 355-373. 
[4] H. H. Chen and M. L. Fang, Shared values and normal families of meromorphic functions, J. Math. Anal. Appl. 260 (2001), 124-132.

[5] G. Frank, Eine Vermutung von Hayman über Nullstellen meromorpher Funktionen, Math. Z. 149 (1976), 29-36.

[6] G. Frank und W. Schwick, Meromorphe Funktionen, die mit einer Ableitung drei Werte teilen, Results Math. 22 (1992), 679-684.

[7] - - - A counterexample to the generalized Bloch principle, New Zealand J. Math. 23 (1994), 121-123.

[8] W. K. Hayman, Picard values of meromorphic functions and their derivatives, Ann. of Math. (2) 70 (1959), 9-42.

[9] - Meromorphic Functions, Clarendon Press, Oxford, 1964.

[10] J. K. Langley, Proof of a conjecture of Hayman concerning $f$ and $f^{\prime \prime}$, J. London Math. Soc. (2) 48 (1993), 500-514.

[11] E. Mues und N. Steinmetz, Meromorphe Funktionen, die mit ihrer Ableitung Werte teilen, Manuscripta Math. 29 (1979), 195-206.

[12] X. C. Pang, Shared values and normal families, Analysis 22 (2002), 175-182.

[13] X. C. Pang and L. Zalcman, Normality and shared values, Ark. Mat. 38 (2000), 171-182.

[14] —, - Normal families and shared values, Bull. London Math. Soc. 32 (2000), 325-331.

[15] W. Schwick, Sharing values and normality, Arch. Math. (Basel) 59 (1992), 50-54.

[16] Y. F. Wang and M. L. Fang, Picard values and normal families of meromorphic functions with multiple zeros, Acta Math. Sinica (N.S.) 14 (1998), 17-26.

[17] Y. Xu, Sharing values and normality criteria, J. Nanjing Univ. Math. Biquart. 15 (1998), 180-185.

[18] —, Normality criteria concerning sharing values, Indian J. Pure Appl. Math. 30 (1999), 287-293.

[19] L. Zalcman, Normal families: new perspectives, Bull. Amer. Math. Soc. 35 (1998), $215-230$.

Department of Mathematics

Nanjing Normal University

Nanjing 210097, P.R. China

E-mail: mlfang@pine.njnu.edu.cn
Department of Mathematics and Statistics

Bar-Ilan University 52900 Ramat-Gan, Israel E-mail: zalcman@macs.biu.ac.il 